\title{
Nanoporous metal tips as frameworks for analysing frozen liquids with atom probe tomography
}

\author{
Levi Tegg, Ingrid McCarroll, Takanori Sato, Matthew Griffith and Julie Cairney
}

The University of Sydney, United States

Atom probe tomography (APT) is a microscopy tool that combines sub-nanometre spatial resolution with chemical information at the part-per-million sensitivity. However, this powerful technique has historically been limited to dense, conductive solids due to the stringent specimen geometry requirements [1-3]. Developing a workflow that enables the analysis of liquids and soft matter is one of the next big frontiers for translating the technique to wider materials science applications [3], and several groups have recently reported progress in this area [4-7]. Examples include the deposition of liquids and polymers onto metal tips [8,9], encapsulating aqueous solutions using graphene [10], using focused ion beam (FIB) milling and lift-out to observe water-glass interfaces $[11,12]$, and using a FIB to mill water which has been cast and frozen on nanoporous gold [13] or microstructured tungsten [14].

We have been investigating the use of nanoporous metal tips as frameworks to support water and aqueous media, an approach that was recently reported to show significant potential [13]. Figure 1 shows a schematic of the proposed workflow and sample geometry. A binary alloy (AB) of a more (A) and less (B) reactive metal is sectioned into matchsticks, then electropolished to a fine tip using conventional methods [2]. The tip is then dealloyed using an acid which attacks metal A but not metal B. The nanoporous tip is then immersed in the sample solution, allowing the liquid to intercalate into the pores, before being plunge-frozen in liquid nitrogen or ethane and transferred to cryogenic vacuum storage [3, 7] for analysis.

The prototypical nanoporous metal is $\mathrm{Au}$ from dealloyed $\mathrm{AgAu}$, but these metals present challenges in electropolishing and in field evaporation, prompting our search for alternatives. We have been investigating the $\mathrm{MnNi}$ system, as nanoporous $\mathrm{Ni}$ can be prepared by etching $\mathrm{Mn}$ with a $\left(\mathrm{NH}_{4}\right)_{2} \mathrm{SO}_{4}$ solution [15]. Figure 2(a) shows a scanning electron microscope image of a nanoporous Ni matchstick produced using this method. However, the use of more reactive metals leads to new unsolved challenges in sample preparation. Figure 2(b-d) shows an X-ray spectroscopy map of a MnNi tip which has been dealloyed, showing the depletion of Mn but with significant oxidation.

It is believed that the nanoporous metal framework will help overcome some of the challenges in analysing soft matter in the atom probe. Specifically, preparing the specimens via electropolishing avoids the complexities of cryo-FIB, and the use of a metallic framework ensures high conductivity at the apex of the tip. In this presentation, we will report on the preparation and characterisation of nanoporous metal tips and discuss our progress in analysing frozen water via atom probe tomography. 


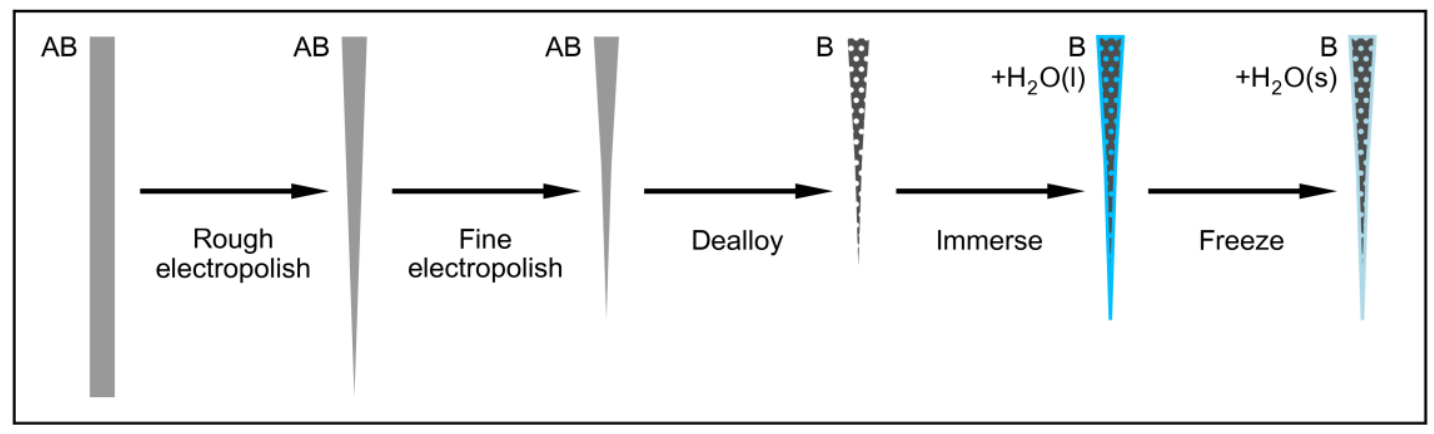

Figure 1. The proposed workflow for the preparation of ice-loaded nanoporous metal tips, showing how the sample geometry and composition changes with each processing step.
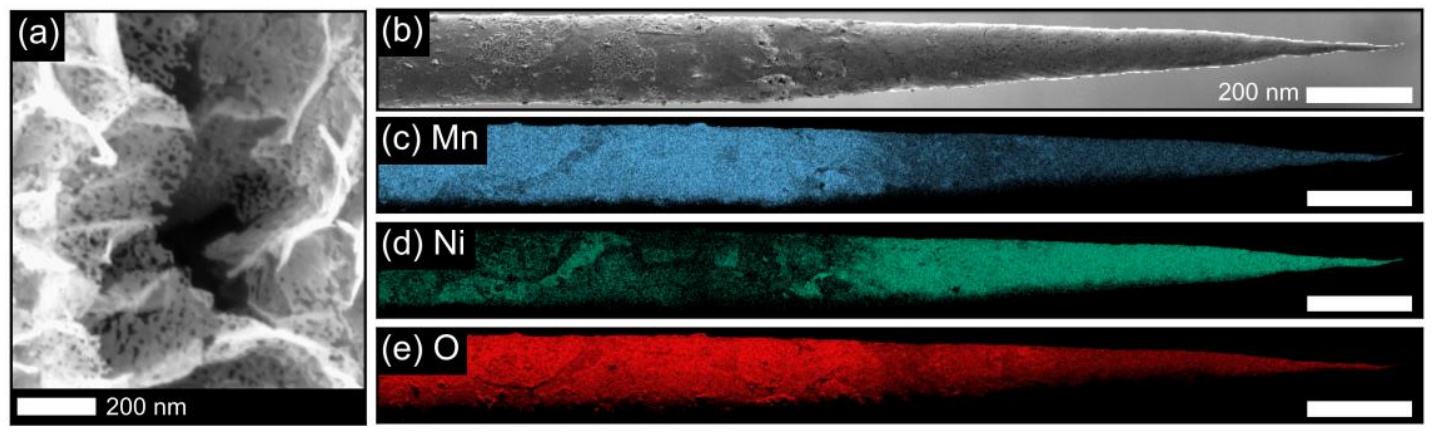

Figure 2. Preparation of nanoporous $\mathrm{Ni}$ from a MnNi alloy. (a) Secondary-electron micrograph of a dealloyed Ni matchstick, showing the nanoporous structure. (b) Secondary-electron micrograph and (c) $\mathrm{Mn},(\mathrm{d}) \mathrm{Ni}$ and (e) $\mathrm{O} \mathrm{K} \alpha \mathrm{X}$-ray intensity maps of a MnNi tip after dealloying.

\section{References}

[1] T. F. Kelly and M. K. Miller. Review of Scientific Instruments 78, 031101 (2007).

[2] B. Gault et al. Atom Probe Microscopy. (Springer, 2012).

[3] I. E. McCarroll et al. Materials Today Advances 7, 100090 (2020).

[4] D. E. Perea et al. Adv Struct Chem Imag 3, 12 (2017).

[5] C. Macauley et al. PLOS ONE 16, e0245555 (2021).

[6] P. Stender et al. arXiv:2102.01954 [cond-mat] (2021).

[7] Y.-S. Chen, M. J. Griffith, and J. M. Cairney. Nano Today 37, 101107 (2021).

[8] T. F. Kelly et al. MRS Bulletin 34, 744-750 (2009).

[9] J. A. Panitz. Microscopy and Microanalysis 14, 122-123 (2008).

[10] S. Qiu et al. Ultramicroscopy 216, 113036 (2020).

[11] D. K. Schreiber, et al. Ultramicroscopy 194, 89-99 (2018).

[12] D. E. Perea et al. npj Materials Degradation 4, 1-7 (2020).

[13] A. A. El-Zoka et al. Science Advances 6, eabd6324 (2020).

[14] T. M. Schwarz et al. Scientific Reports 10, 20271 (2020).

[15] H.-J. Qiu et al. Journal of Power Sources 247, 896-905 (2014)., 896-905 (2014). 